\title{
Degradable mesoporous semimetal antimony nanospheres for near-infrared II multimodal theranostics
}

\section{Yu Chen}

Shenzhen University

\section{Zhongzheng Yu}

Nanyang Technological University

\section{Yaguang Ren}

Shenzhen Institutes of Advanced Technology

\section{Kai Zheng}

Northwestern Polytechnical University

Meng Wang

Shenzhen University

\section{Qiang Wu}

Shenzhen university

\section{Feifan Zhou}

Shenzhen university

\section{Chengbo Liu}

Shenzhen Institutes of Advanced Technology, Chinese Academy of Sciences https://orcid.org/00000001-7565-2044

\section{Liwei Liu}

Shenzhen University

\section{Jun Song}

Shenzhen University https://orcid.org/0000-0002-2321-7064

Junle Qu ( $\sim$ jlqu@szu.edu.cn )

Shenzhen University https://orcid.org/0000-0001-7833-4711

\section{Article}

Keywords: Mesoporous Antimony, Photodegradable, Photothermal therapy, Photoacoustic imaging

Posted Date: May 27th, 2021

DOI: https://doi.org/10.21203/rs.3.rs-521968/v1 
License: (c) (i) This work is licensed under a Creative Commons Attribution 4.0 International License. Read Full License

Version of Record: A version of this preprint was published at Nature Communications on January 27th, 2022. See the published version at https://doi.org/10.1038/s41467-021-27835-y. 


\section{Abstract}

Metallic and semimetallic mesoporous frameworks are of great importance owing to their unique properties and broad applications. However, semimetallic mesoporous structures cannot be obtained by the traditional template-mediated strategies due to the inevitable hydrolytic reaction of semimetal compounds. Therefore, it is yet challenging to fabricate mesoporous semimetal nanostructures, not even mention controlling their pore sizes. Thus, we have developed a facile and robust selective etching route to synthesize monodispersed mesoporous antimony nanospheres (MSbNSs). This is the first report of semimetallic mesoporous structure to the best of our knowledge. The pore sizes of MSbNSs are tunable by carefully controlling the partial oxidation of $\mathrm{Sb}$ nuclei and the selective etching of the as-formed $\mathrm{Sb}_{2} \mathrm{O}_{3}$. MSbNSs showed a wide absorption from visible to second near-infrared (NIR-II) region. Moreover, PEGylated MSbNSs were degradable and the degradation mechanism was further explained. The NIR-II photothermal performance of MSbNSs was excellent with a high photothermal conversion efficiency of $\sim 44 \%$ and intensive NIR-II photoacoustic signal. MSbNSs showed great potential as multifunctional nanomedicines for NIR-Il photoacoustic imaging guided synergistic photothermal/chemo therapy in vivo. Our novel selective etching process would contribute to the development of various semimetallic mesoporous structures and efficient multimodal nanoplatforms for theranostics.

\section{Introduction}

Mesoporous nanomaterials with large surface areas, tunable pore sizes, and diverse compositions have drawn tremendous interest due to their great potential in various applications, including drug delivery, catalysis, energy conversion and storage, as well as separation technique. ${ }^{1,2,3,4,5,6}$ In the past two decades, numerous silica-based mesoporous structures with tunable pore sizes and morphologies were constructed by a modified stöber process, $7,8,9,10,11,12,13$ but the inert properties of silica seriously limited their applications. Therefore, metallic and semimetallic mesoporous frameworks are expected to show superior performance than silica-based mesoporous structures in electronics, catalysis, optics, and biophotonics, owing to the extraordinary electronic properties of metals and semimetals. ${ }^{14,15,16,17}$ Several metallic mesoporous nanostructures, including platinum, palladium, gold and rhodium etc. have been successfully prepared in recent years. ${ }^{18,19,20,21,22}$ The synthesis method for metallic mesoporous nanostructures were currently limited to templated-mediated method or wet chemical method. ${ }^{23}$ But the formation mechanism and controllable synthesis of these metallic mesoporous structures accompanying with demonstrations in various applications have attracted huge attention. ${ }^{24,25,26}$ In contrast, semimetallic mesoporous structures have rarely been reported. ${ }^{27}$ We attribute the main obstacle to the inevitable hydrolytic reaction and rapid precipitation of semimetal compounds (arsenic, antimony and bismuth) that result in the lack of necessary interaction between the semimetal precursor and poreforming template. Hence, a facile and robust approach for the controllable synthesis of semimetallic mesoporous nanomaterials is still in urgent need and of great challenge. 
Furthermore, semimetals are reported to show decent photothermal performance in the second nearinfrared (NIR-II, 1000-1700 nm) range. 28, 29, 30, 31 Semimetal-based photothermal agents (PTAs) can absorb the energy of NIR-II light and convert it to heat for photothermal therapy (PTT), and simultaneously generate photoacoustic signals for deep-tissue photoacoustic imaging (PAl). ${ }^{32,33,34,35,36}$ Semimetallic mesoporous nanomaterials are excellent candidates for multimodal theranostic agents with loading capacity and photothermal conversion ability. Recently, antimony (Sb) nanomaterials have been demonstrated to have a wide absorption range from visible to NIR-II region, remarkable photothermal conversion efficiency (PTCE) and intensive photoacoustic signal in the first near-infrared (NIR-I, 700-1000 $\mathrm{nm}$ ) region. ${ }^{37,38,39,40}$ There are few studies of Sb-based PTAs in the NIR-II range. Furthermore, Sb nanomaterials have shown the photothermal induced degradable property, which facilitated the fast clearance of $\mathrm{Sb}$ nanomaterials to avoid the long-term toxicity issue of nanomedicines. Therefore, mesoporous Sb-based nanoconstructs with tunable pore sizes are expected to achieve NIR-II PAl guided synergistic therapy with safer and better therapeutic outcomes.

Herein, we describe a novel and effective route to prepare the monodispersed mesoporous $\mathrm{Sb}$ nanospheres (MSbNSs) with tunable pore sizes via a selective etching process. We systemically studied the formation mechanism of MSbNSs and realized the controllable tuning of MSbNSs pore sizes. The resultant MSbNSs have been proven to possess a high NIR-II PTCE up to $44 \%$, intensive NIR-II photoacoustic signal, and photothermal-induced degradation. The photothermal degradation mechanism of MSbNSs has been proposed, simulated and analyzed. Based on these findings, MSbNSs-based multifunctional nanomedicines were successfully constructed and presented for NIR-II PAI guided synergistic photothermal/chemo therapy in vivo. The PEGylated MSbNSs loaded with doxorubicin (DOX) showed excellent drug loading and release ability and excellent NIR-II photothermal performance with elimination of tumors in mice. Our approach of selective etching would contribute to the development of more types of semimetallic mesoporous nanostructures and more multimodal theranostics by loading different drugs, antigens or mRNAs for multifunctional chemotherapy, immunotherapy or gene therapy with NIR-II guided PAI and PTT.

\section{Results}

\section{Preparation, characterization and structural control of MSbNSs.}

The schematic illustration of the formation process of MSbNSs is shown in Fig. 1a. Dodecylthiol (DDT) and oleylamine (OLA) were used as capping ligands to control the shape of Sb nanospheres, while octadecene (ODE) was used as solvent, and $\mathrm{SbCl}_{3}$ and tert-butylamine borane were selected as semimetal precursor and reducing agent, respectively. After chemical reduction, numerous small $\mathrm{Sb}$ nuclei were formed, which were sensitive to oxygen and could be easily oxidized due to their high surface energy. As the $\mathrm{Sb}$ nanocrystals grew, the oxidation product antimony oxide $\left(\mathrm{Sb}_{2} \mathrm{O}_{3}\right)$ was dispersed in $\mathrm{Sb}$ nanocrystals, which could further react with DDT in the solution as the selective etching process of $\mathrm{Sb}_{2} \mathrm{O}_{3}$ to form mesopores. The pore sizes of MSbNSs are tunable by carefully controlling the oxidative degree of 
$\mathrm{Sb}$ nuclei and the selective etching of the as-formed $\mathrm{Sb}_{2} \mathrm{O}_{3}$. Detailed experimental procedures were provided in experimental section. The resultant MSbNSs have shown uniform spherical morphologies in the transmission electron microscopy (TEM, Supplementary Fig. 1), and their high-angle annular darkfield scanning TEM (HADDF STEM) images (denoted as MSbNSs-1, MSbNSs-2, MSbNSs-3 and MSbNSs4) were shown in Fig. $1 \mathrm{~b}$-e. The size of MSbNSs were gradually increased from $45 \mathrm{~nm}$, to $48 \mathrm{~nm}, 51 \mathrm{~nm}$, and to final $58 \mathrm{~nm}$ (Supplementary Fig. 2), respectively. $\mathrm{N}_{2}$ absorption and pore size analysis was performed to demonstrate the mesoporous structures of MSbNSs (Fig. 1f). The obvious hysteresis loop of the absorption/desorption isotherms in all of the cases implied the typical mesoporous structures of the resultant MSbNSs. The Brunauer-Emmett-Teller (BET) surface area of MSbNSs increased from 78 $\mathrm{m}^{2} \mathrm{~g}^{-1}$ to $231 \mathrm{~m}^{2} \mathrm{~g}^{-1}, 235 \mathrm{~m}^{2} \mathrm{~g}^{-1}$ and to final $234 \mathrm{~m}^{2} \mathrm{~g}^{-1}$. MSbNSs with different pore sizes were obtained via selective etching of SbNSs by controlling the amount of oxygen $\left(\mathrm{O}_{2}\right)$ in the reaction precursor. By controlling the pumping time of $\mathrm{O}_{2}$ from 10 s to 30 s, the mesopore size of MSbNSs has been increased from 2.6 to $3.7 \mathrm{~nm}$. Increase of $\mathrm{O}_{2}$ amount has generated larger pores $(\sim 11.5 \mathrm{~nm})$ in MSbNSs-3 besides small mesopores with average sizes of $3.9 \mathrm{~nm}$ and $5.7 \mathrm{~nm}$. The nitrogen sorption isotherms of the MSbNSs-3 showed two major capillary condensation steps in the relative pressure ranges 0.1-0.3 and $0.75-0.98$, respectively, indicating that at least two sets of pores coexisted in the nanospheres. MSbNSs-1, MSbNSs-2 and MSbNSs-3 can still remain integrated spherical shape. Nevertheless, further increased $\mathrm{O}_{2}$ amount would destroy the nanostructure of MSbNSs and lead to collapsed MSbNSs-4 with larger pores $(\sim 18 \mathrm{~nm})$. To further confirm the important role of $\mathrm{O}_{2}$ in the formation of mesopores, a control experiment without the pumping of $\mathrm{O}_{2}$ was performed under the situation that other experimental parameters were kept constant. Mesoporous structures were not observed as shown in Supplementary Fig. 3. MSbNSs with tunable mesopore sizes were successfully fabricated by our selective etching method for the first time to the best of our knowledge. The shape of MSbNSs can also be facilely tuned by regulating the reaction temperature (Supplementary Fig. 4).

Moreover, MSbNSs-1, MSbNSs-2 and MSbNSs-3 showed strong and broad absorption, especially in the NIR-II range (Fig. 1g). From MSbNSs-1 to MSbNSs-3, the absorption peaks were further red-shifted towards NIR-II range, providing the potential for excellent NIR-II photothermal performance. The collapsed MSbNSs-4 lost the strong NIR-II absorption, which is not applicable for the multimodal theranostics.

\section{Formation mechanism of MSbNSs.}

To shed light on the novel selective etching mechanism, X-ray diffraction (XRD) and X-ray photoelectron spectroscopy (XPS) were carried out to analyze the reaction process. XRD patterns revealed that MSbNSs-1 and MSbNSs-2 remained pure rhombohedral phase of Sb corresponding with the standard card JCPDS 085-1324, while a mixed phase of rhombohedral phase of $\mathrm{Sb}$ and cubic phase of $\mathrm{Sb}_{2} \mathrm{O}_{3}$ corresponding with JCPDS 05-0534 were detected in MSbNSs-3 and MSbNSs-4 (Fig. 2a). XPS results further illustrated that lower amount of $\mathrm{O}_{2}$ first oxidized $\mathrm{Sb}$ to form $\mathrm{Sb}_{2} \mathrm{O}_{3}$ as measured in MSbNSs-1, while higher amount of $\mathrm{O}_{2}$ would further generate $\mathrm{Sb}_{2} \mathrm{~S}_{3}$ that derived from the reacting $\mathrm{Sb}_{2} \mathrm{O}_{3}$ with DDT, since $\mathrm{Sb}_{2} \mathrm{~S}_{3}$ first appeared in MSbNSs-2 and gradually increased in MSbNSs-3 and MSbNSs-4 (Fig. 2b). 
$\mathrm{Sb}_{2} \mathrm{O}_{3}$ and $\mathrm{Sb}_{2} \mathrm{~S}_{3}$ generated in the early oxidation process were not crystalized and $\mathrm{Sb}_{2} \mathrm{~S}_{3}$ can be dissolved in reaction solvent to form the mesoporous structures, thus not detectable in XRD patterns. Moreover, $\mathrm{Sb}_{2} \mathrm{~S}_{3}$ could react with $\mathrm{O}_{2}$ to form $\mathrm{Sb}_{2} \mathrm{O}_{3}$ and $\mathrm{SO}_{2}$, leading to a dynamic reaction cycle to accelerate the etching process. A higher amount of $\mathrm{O}_{2}$ could even further oxidize $\mathrm{Sb}_{2} \mathrm{O}_{3}$ to $\mathrm{Sb}_{2} \mathrm{O}_{5}$ as $\mathrm{Sb}_{3} \mathrm{~d}_{5 / 2}$ only appeared in MSbNSs-4. Therefore, we proposed a step-oxidization reaction mechanism to form the mesoporous structure in the following three steps as shown in Fig. 2c.

$\mathrm{Sb}+\mathrm{O}_{2} \rightarrow \mathrm{Sb}_{2} \mathrm{O}_{3}$

$\mathrm{Sb}_{2} \mathrm{O}_{3}+\mathrm{C}_{12} \mathrm{H}_{25} \mathrm{SH} \rightarrow \mathrm{Sb}_{2} \mathrm{~S}_{3}+\mathrm{C}_{12} \mathrm{H}_{25} \mathrm{OH}$

$\mathrm{Sb}_{2} \mathrm{~S}_{3}+\mathrm{O}_{2} \rightarrow \mathrm{Sb}_{2} \mathrm{O}_{3}+\mathrm{SO}_{2}$

$\mathrm{Sb}_{2} \mathrm{O}_{3}+\mathrm{O}_{2} \rightarrow \mathrm{Sb}_{2} \mathrm{O}_{5}$

Step (2) and Step (3) were further confirmed by measuring the Fourier-transform infrared spectroscopy (FTIR) spectra of the reaction solutions (Fig. 2d). O-H stretching of alcohol $\left(3550-3200 \mathrm{~cm}^{-1}\right)$ and S=O stretching of $\mathrm{SO}_{2}\left(1350-1300 \mathrm{~cm}^{-1}\right)$ were tested to be stronger as the increase of $\mathrm{O}_{2}$ amount, especially in the solvent of MSbNSs-4. Elemental mapping using energy-dispersive X-ray spectroscopy (EDS) under STEM mode further confirmed the existence of $O$ and $S$ in MSbNSs (Fig. 2e, Supplementary Fig. 5). The corresponding mass percentages of $\mathrm{Sb}, \mathrm{O}$ and $\mathrm{S}$ elements in MSbNSs-3 are $94.5 \%, 3.3 \%$ and $2.2 \%$, respectively (Supplementary Fig. 6). From MSbNSs-2 to MSbNSs-4, although the amount of $\mathrm{O}_{2}$ in the reaction precursor were increasing, the residual ratios of $\mathrm{O}$ elements in the final products were decreasing, while the ratios of S element were increasing with a significant increase in MSbNSs-4, which further proved our proposed reaction mechanism (Fig. 2f).

\section{NIR-II photothermal, degradation and drug loading/release performance of MSbNSs.}

Different from the good photothermal stability of $\mathrm{Sb}$ nanopolyhedrons reported in our previous work, ${ }^{37}$ the as-synthesized MSbNSs showed varying degrees of photothermal degradation under $1210 \mathrm{~nm}$ laser irradiation (Fig. 3a). In particular, MSbNSs-3 showed the most obvious photothermal degradation among MSbNSs-1/2/3. The detailed degradation behavior of MSbNSs-3 was first studied by TEM (Fig. 3b). Obvious degradation of the mesopores in MSbNSs-3 and numerous soluble Sb species could be observed after two cycles of irradiation. Almost all the spherical structures were destroyed after three successive cycles of irradiation, indicating that MSbNSs-3 gradually collapsed after laser irradiation. The color of the black MSbNSs-3 solution also gradually faded to transparent after three cycles of irradiation. The photothermal stability of MSbNSs-2 decreased to some extent, but the degradation of MSbNSs-2 proceeded slowly (Supplementary Fig. 7). The degradation of the mesopores in MSbNSs-2 can also be observed after three successive cycles of irradiation, but the collapse of mesoporous nanostructures would not appear. By comparison, MSbNSs-1 showed the best photothermal stability (Supplementary Fig. 8). The photothermal degradation mechanism of MSbNSs was also analyzed based on the FDTD 
simulations as Sb-based nanostructures possess localized surface plasmon resonance (LSPR) effect. ${ }^{41}$ The detailed fitting process is described as shown in supporting information. The results showed that electric fields at the joints and gaps inside the mesoporous structure were amplified by more than several orders of magnitude, resulting in stronger localization of heat generation in mesopores. The simulation results indicated that the mesopores of both MSbNSs-2 (Fig. 3c) and MSbNSs-3 (Fig. 3d) could generate localized "hot channels" and similar heat power densities were generated in MSbNSs-2 and MSbNSs-3, while "hot channels" would gradually disappear as mesopores grew larger $(\sim 11.5 \mathrm{~nm})$, which is consistent with the change of the photothermal performance of MSbNSs-2 and MSbNSs-3. During the photothermal process, the increased temperature would promote the following reaction (Step (5)) of surface $\mathrm{Sb}$ atoms with water molecules to form $\mathrm{Sb}-\mathrm{H}$ and $\mathrm{Sb}-\mathrm{OH}$, leading to the dissolution of $\mathrm{Sb}$ nanocrystals in aqueous solution. ${ }^{42}$

$\mathrm{Sb}+2 \mathrm{H}_{2} \mathrm{O} \rightarrow \mathrm{Sb}-\mathrm{OH}_{2}+\mathrm{Sb}-\mathrm{HOH} \rightarrow \mathrm{Sb}-\mathrm{H}+\mathrm{Sb}-\mathrm{OH}$

Since MSbNSs-3 have larger pore sizes, the amount of accessible water within the NSs would be higher. The photothermal degradation process was further promoted in MSbNSs-3. The looser mesoporous structure and lower crystallinity of MSbNSs-3 could also accelerate the dissolution and result in the enhanced photothermal degradation property in MSbNSs-3.

The photothermal degradation property of MSbNSs can further boost the release efficiency as nanocarriers for different types of drugs. We evaluated their capabilities of drug delivery and NIR-II triggered on-demand release of drugs using DOX as a typical anticancer model drug (Fig. 3e). The drug loading capacity and efficiency and of MSbNSs-2 and MSbNs-3 were studied as shown in Fig. 3f. A burst in the release of DOX ( 53\%) can be observed with $1210 \mathrm{~nm}$ irradiation, which is about 6 times higher than that of the group without NIR-II light irradiation (Fig. 3g). The on-demand release properties under NIR-II laser irradiation could be ascribed to the collapse of the MSbNSs and local photothermal effect to accelerate the diffusion of drugs. All of the above results reveal that the MSbNSs can be used simultaneously as agents for PTT, PAI and novel drug carriers for NIR-Il controlled drug release.

Considering the appropriate pore size and NIR-II absorption wavelength as well as photothermal degradation, we have chosen MSbNSs-3 to perform multimodal theranostics in the following work. MSbNSs-3 were PEGylated and the absorption properties of PEGylated MSbNSs-3 in aqueous solution still exhibited broad absorption from visible to NIR wavelengths and the strongest absorption peak was $1480 \mathrm{~nm}$, located within the NIR-II window (Fig. 4a). The strong absorption of PEGylated MSbNSs-3 in the NIR-II region suggest their potential in NIR-II PAI and PTT. The photothermal effect of PEGylated MSbNSs3 was studied by the irradiation of $1210 \mathrm{~nm}$ laser. This provides another efficient NIR-II excitation wavelength besides the typical $1064 \mathrm{~nm}$. As shown in Fig. 4b, 4c, the temperature variation of PEGylated MSbNSs-3 displayed a concentration-dependent behavior. We also investigated the photothermal performances of PEGylated MSbNSs-3 irradiated under different power densities (Fig. 4d). The PTCE of PEGylated MSbNSs-3 was calculated to be $~ 39 \%$ (Fig. 4e), which is superior to the majority of current NIR-II PTAs. By comparison, PEGylated MSbNsS-1 and MSbNSs-2 was calculated to be $~ 44 \%$ and $~ 41 \%$ 
(Supplementary Fig. 9). Moreover, a strong concentration-related photoacoustic signal of PEGylated MSbNSs-3 was observed (Fig. 4f), implying that PEGylated MSbNSs-3 could be used as bright NIR-II photoacoustic contrast agents.

\section{In vitro synergistic PTT and chemotherapy.}

To study the synergistic therapeutic outcomes of DOX-loaded PEGylated MSbNSs-3 (PEGylated MSbNSs3/DOX), the cellular internalization process was first checked in panc02 cancer cells using confocal microscopy. The fluorescence of DOX overlapped with the fluorescence of Lyso-tracker (Fig. 5a), indicating that the uptake process was mainly achieved by endocytosis. The cytotoxicity and treatment efficacy of PEGylated MSbNSs-3/DOX were further tested by Live/dead assays. PEGylated MSbNSs-3 with/without DOX were incubated with panc02 cancer cells under different concentrations from 0 to 100 $\mu \mathrm{g} \mathrm{mL} \mathrm{m}^{-1}$ (Fig. 5b). Without $1210 \mathrm{~nm}$ irradiation, PEGylated MSbNSs-3 showed a high cell viability indicating a negligible cytotoxicity, meanwhile PEGylated MSbNSs-3/DOX would kill cancer cells by releasing DOX. The in vitro chemotherapy efficacy is limited compared with NIR-II PTT. The $1210 \mathrm{~nm}$ irradiation on PEGylated MSbNSs-3 could generate heat to kill cancer cells, leading to a cell viability reduced to $20 \%$ at $100 \mu \mathrm{g} \mathrm{mL}^{-1}$. The synergistic PTT and chemotherapy showed an improved in vitro therapeutic capability with a minimal cell viability of $8 \%$ (Supplementary Fig 10 ).

\section{In vivo NIR-II PAl-guided PTT and chemotherapy.}

In vivo $1210 \mathrm{~nm}$ PAI was conducted to guide the optimal systemic administration window of PEGylated MSbNSs-3/DOX. The PA signal in the tumor increased gradually after the intravenous injection of PEGylated MSbNSs-3/DOX, indicating the gradual accumulation of these nanomedicines in the tumor site (Fig. 5c). The PA signal achieved the highest at $8 \mathrm{~h}$ post-injection, with a 9-fold higher PA amplitude than the pre-injection tumor (Supplementary Fig.11). With the guidance of PAl, the photoirradiation of $1210 \mathrm{~nm}$ was conducted at $8 \mathrm{~h}$ post-injection to prove the therapeutic efficacy of the synergistic NIR-II PTT and chemotherapy on 4T1 tumor-bearing mice. The tumor temperature of PEGylated MSbNSs$3 / \mathrm{DOX}$ treated mice increased rapidly to $\sim 46{ }^{\circ} \mathrm{C}$ within $3 \mathrm{~min}$ and finally arrived to $\sim 50{ }^{\circ} \mathrm{C}$ at $7 \mathrm{~min}$, which is significantly higher than that of PBS-treated tumors (Fig. $5 \mathrm{~d}$ ). To further investigate the in vivo photothermal triggered degradation of PEGylated MSbNSs-3, PA images before and after laser irradiation were studied as shown in Fig.5e. An obvious photothermal degradation of PEGylated MSbNSs-3 was observed after the irradiation of $1210 \mathrm{~nm}$ laser for $10 \mathrm{~min}$, which was indicated by the dramatic drop of PA intensity of tumor by $70 \%$ (Supplementary Fig. 12). To assess the clearance of PEGylated MSbNSs-3, the biodistribution of MSbNSs-3 in mice were studied. After the PEGylated MSbNSs-3 were injected into mice and without irradiation of $1210 \mathrm{~nm}$ laser, the distribution of PEGylated MSbNSs-3 in major organs were measured at 1, 7, 14 days. PEGylated MSbNSs-3 were mainly cumulated in the liver, spleen and kidney (Fig. 5f).

The tumor sizes in different groups were measured and compared (Fig. $5 \mathrm{~g}$ ). Mono chemotherapy by PEGylated MSbNSs-3/DOX showed a mild tumor inhibition rate. Meanwhile, the NIR-II PTT by PEGylated 
MSbNSs-3 showed excellent therapeutic outcomes with eradication of tumors owing to the excellent photothermal performance of MSbNSs-3. Tumors treated by PEGylated MSbNSs-3/DOX for synergistic NIR-II PTT and chemotherapy were also eradicated, with an even faster speed. The body weights of mice in all the groups were not influenced by different treatments in all the groups (Fig. 5h). The survival rates of mice were monitored to further prove the treatment efficacy. The treatment of NIR-II PTT and chemotherapy resulted in a highest survival rate (Fig. 5i). Moreover, there were also no obvious histopathological abnormalities found in major organs of the mice (Supplementary Fig. 13), indicating the good biocompatibility of the MSbNSs nanoplatforms. Hematoxylin and eosin (H\&E) staining and TdTmediated Dutp-biotin nick end-labeling (TUNEL) staining of tumor slices evaluated the necrosis and apoptosis of tumor cells in all the groups (Fig. 5j). No apparent necrosis or apoptosis was found in control groups, PEGylated MSbNSs-3/DOX caused moderate necrosis and apoptosis by releasing DOX. Large areas of necrosis and apoptosis were found in $1210 \mathrm{~nm}$ irradiated PEGylated MSbNSs-3 and PEGylated MSbNSs-3/DOX groups. The synergistic NIR-II PTT and chemotherapy caused the most necrosis and especially apoptosis, which is also consistent with the tumor growth curves. Altogether, these data demonstrated that our novel PEGylated MSbNSs-3/DOX nanoplatform showed high potential for multimodal NIR-II theranostics with excellent therapeutic efficacy, good biocompatibility and photothermal degradability.

\section{Discussion}

In summary, we developed a novel selective etching method to fabricate semimetallic mesoporous nanostructures for the first time to the best of our knowledge. By controlling the oxidative degree of $\mathrm{Sb}$ nuclei, the as-synthesized MSbNSs possessed mesopores with tunable pore sizes. The detailed selective etching reaction processes and mechanism were further explored and explained. Moreover, MSbNSs also demonstrated excellent NIR-II photothermal performance, showing a strong NIR-II PAI contrast and relatively high NIR-II PTCE ( 44\%). Thus, MSbNSs have shown great potential as integrated nanoplatforms for NIR-II PAl guided synergistic chemotherapy and NIR-II PTT. We also observed that the enhanced photothermal degradability of MSbNSs as the sizes of mesopores increases. The FDTD calculation results revealed that the etched mesopores would lead to localized "hot" channels within the mesopores, which would accelerate the degradation of $\mathrm{Sb}$ nanostructures. Larger pore sizes would lead to a higher amount of water within the MSbNSs, looser structure and lower crystallinity, leading to a faster photothermal degradation.

With the guidance of in vivo NIR-II PAI, the synergistic NIR-II PTT and chemotherapy by PEGylated MSbNSs-3/DOX showed excellent therapeutic outcomes with tumor elimination and highest posttreatment survival rate. The multimodal theranostic nanoplatform could also provide various combinations of NIR-II PAI and PTT with other therapeutic modalities via loading various components. Our selective etching method can be widely applied in different material systems, including 2D materials, multilayer structures and composite materials etc. utilizing semimetals for enhanced performance and multifunctionality. 


\section{Methods}

Synthesis of MSbNSs with tunable pore sizes. In a typical synthesis of MSbNSs, $1 \mathrm{~mL}$ Dodecylthiol (DDT), $5 \mathrm{~mL}$ Oleylamine (OLA), $3 \mathrm{~mL}$ octadecene (ODE) and antimony trichloride $\left(\mathrm{SbCl}_{3}\right)(0.05 \mathrm{~g}$, $0.22 \mathrm{mmol}$ ) were sequentially added in a $50 \mathrm{~mL}$ three necked flask. Then the reaction mixture was stirred and degassed under vacuum at $50{ }^{\circ} \mathrm{C}$ until the $\mathrm{SbCl}_{3}$ dissolved completely in solvent. Next, the flask was heated to $170{ }^{\circ} \mathrm{C}$. On the other hand, tert-Butylamine borane $(0.03 \mathrm{~g}, 0.35 \mathrm{mmol})$ was dissolved completely in $1 \mathrm{~mL}$ of OLA. Then, $1 \mathrm{~mL}$ solution of tert-Butylamine borane in OLA was injected swiftly to the reaction system, simultaneously the pumping time of $\mathrm{O}_{2}$ from $1 \mathrm{~s} / 10 \mathrm{~s} / 30 \mathrm{~s}$ was performed for controlling the amount of $\mathrm{O}_{2}$ in the reaction precursor. After stirring for $30 \mathrm{~min}$, the reaction was stopped by immersing the flask into the ice-water bath. At $50{ }^{\circ} \mathrm{C}$, dried $\mathrm{OA}(0.1 \mathrm{~mL})$ was added to displace weakly bound OLA ligand capping. Sb NPHs were precipitated by ethanol $15 \mathrm{~mL}$, separated by centrifugation, redispersed in toluene $(5 \mathrm{~mL})$ containing $\mathrm{OA}(0.1 \mathrm{~mL})$. The samples were sealed and kept in dark before using. The morphology of MSbNSs can be tuned by adjusting the mole of $\mathrm{SbCl}_{3}$ and mole ratio of DDT/OLA.

Mouse Tumor Model. All of our animal experiments were conducted in compliance with the guidelines established by the Animal Ethical and Welfare Committee of Shenzhen University (AEWC-SZU). For photothermal therapy, the tumors were established on female C57BL/ 6 mice by subcutaneous injection of two million panc 02 cells suspended in $0.2 \mathrm{~mL}$ of DMEM ( $10 \% \mathrm{FBS}, 1 \%$ antibiotics) into side of the back of mice. Tumors were allowed to grow until a single aspect was longer than $6 \mathrm{~mm}$ before being used for photothermal treatment.

Synthesis of PEGylated MSbNSs. The as-synthesized MSbNSs were dispersed in dichloromethane (5 mL, $4 \mathrm{mg} \mathrm{mL}^{-1}$ ), followed by mixing with 1,2-distearoyl-sn-glycero-3-phosphoethanolamineN[amino(polyethylene glycol)2000] (DSPE-PEG2K) at concentrations at $4 \mathrm{mg} \mathrm{mL}^{-1}$. Subsequently, the resulting mixture was sonicated for about $30 \mathrm{~min}$, and the solvent of dichloromethane was fully removed via rotary evaporator. The residual solid was redispersed by adding $4 \mathrm{~mL}$ purified water and sonicated for $30 \mathrm{~min}$. The resulting products were collected by centrifugation $10000 \mathrm{rpm}$ for $5 \mathrm{~min}$, washed with purified water three times, and redispersed in $4 \mathrm{~mL}$ PBS. The samples were sealed and kept in dark before using.

DOX loading and releasing. DOX were added into the resultant PEGylated MSbNSs-3 solution ( $1 \mathrm{mg} \mathrm{mL}^{-}$ $\left.{ }^{1}\right)$. After magnetically stirring for $24 \mathrm{~h}$, unloaded drugs were removed by centrifugation at $8000 \mathrm{rpm}$. To determine the drug loading capacity and efficiency of the nanoparticles. Different concentrations of DOX (0 0.8 mg/mL of DOX) were stirred with PEGylated MSbNSs-3 $(0.2 \mathrm{mg} / \mathrm{mL})$ for 24 at room temperature. The resultant product was collected by centrifugation at $8000 \mathrm{rpm}$. The amount of unbound DOX in the solution was determined by measuring the absorbance at $488 \mathrm{~nm}$ using a calibration curve prepared under the same condition. The drug loading capacity is defined as (weight of drug loaded on PEGylated 
MSbNSs-3 / weight of PEGylated MSbNSs-3). The drug loading efficiency (\%) is defined as (weight of drug loaded on PEGylated MSbNSs-3 / weight of initially added DOX) $\times 100$.

Cellular uptake. The cellular uptake of the PEGylated MSbNSs/DOX was observed using a confocal laser scanning microscope. In brief, 4T1 cells were seeded into 8-well chambered slides (Thermo Scientific, USA) and cultured for $12 \mathrm{~h}$. Then, the cells were incubated with PEGylated MSbNSs/DOX for 3 hours, and followed with Lyso-Tracker (green color) and Hoechst 33342 (blue color) double staining. The cells were washed with PBS for three times and analyzed via confocal laser scanning microscope.

In vitro phototoxicity of PEGylated MSbNSs. CCK-8 assay was used to evaluate the phototoxicity of PEGylated MSbNSs. Briefly, panc 02 cells were seeded into a 96-well plate with the concentration of $5 \times$ $10^{3}$ cells per well and cultured for $12 \mathrm{~h}$. Then, the medium were replaced with serum-free medium containing PEGylated MSbNSs-3, PEGylated MSbNSs-3/DOX of various concentrations for another $16 \mathrm{~h}$, respectively. Then, each well was washed three time with PBS to remove free MSbNSs. The cells were submitted to $1210 \mathrm{~nm}$ laser irradiation at $0.75 \mathrm{~W} \mathrm{~cm}^{-2}$ for $5 \mathrm{~min}$ and then culture medium was added to each well to incubate for $24 \mathrm{~h}$. The amount of an orange formazan dye, produced by the reduction of WST-8 (active gradient in CCK-8) by dehydrogenases in living cells, is directly proportional to the quantity of living cells in the well. Therefore, by measuring the absorbance of each well at $450 \mathrm{~nm}$ using a microplate reader, cell viability could be determined by calculating the ratio of absorbance of experimental wells to that of the control cell wells.

PA imaging. An optical parametric oscillator laser source (Innolas $\mathrm{GmbH}$, Bonn, Germany) emitting 8 ns pulsed lasers was coupled to a multimode optical fiber with a $1500 \mu \mathrm{m}$ core diameter for the photoacoustic signal excitation. The use of this nanosecond pulsed laser on the tumor tissue resulted in very small thermal expansions. This led to the vibration of the tissue, which generated ultrasonic waves that could be recorded by a commercial 128-element linear array transrectal ultrasound transducer. The Q-switch output of the laser source was synchronized with a Vantage 128 research ultrasound platform (Verasonics, Inc. Kirkland WA, USA) to perform photoacoustic and ultrasound data acquisition. Photoacoustic signals were acquired at a frame rate equal to the laser pulse firing frequency of $30 \mathrm{~Hz}$, and the conventional delay and sum (DAS) reconstruction algorithm was used to reconstruct the photoacoustic B-scan image. The system can simultaneously display both photoacoustic and ultrasound images in real-time.

In vivo Photothermal therapy of PEGylated MSbNSs. BALB/c mice received subcutaneous injection of $5 \times 10^{5} 4 \mathrm{~T} 1$ cells at the right flank. After tumor volume reached $100-150 \mathrm{~mm}^{3}$, mice were randomly divided into 5 groups (5 mice per group): PBS, PBS+L, PEGylated MSbNSs-3/DOX, PEGylated MSbNSs$3+\mathrm{L}$, and PEGylated MSbNSs/DOX $+\mathrm{L}$. At $8 \mathrm{~h}$ post-injection, the tumor regions of living mice were exposed to a $1210 \mathrm{~nm}$ laser with a power density of $1 \mathrm{~W} / \mathrm{cm}^{2}$, using an optical fiber with a diffusion lens (Pioneer Optics, Bloomfield, CT) to delivery uniform light distribution on the treatment surface. The temperature on the tumor surface during PTT was measured with an infrared thermal camera E80 (FLIR, Boston, USA). After photothermal treatment, sizes of tumors were measured by a caliper every other day for 18 days. 
Tumor volume was calculated as follows:volume $=$ (tumor length $) \times(\text { tumor width })^{2} / 2$. One day after treatment, tumors were collected from selected mice in each group were collected for tissue section and H\&E staining.

Characterizations. TEM and STEM observations were taken on a FEI Titan Cubed Themis G2300 transmission electron microscope with an acceleration voltage of $300 \mathrm{Kv}$. Carbon coated copper grids were dipped in the chloroform solutions to deposit the samples onto the films. UV-visible spectra were recorded on an HP8453 UV-visible spectrophotometer. X-ray powder diffraction (XRD) patterns were obtained by using a Philips pw1830 X-ray diffractometer. X-ray photoelectron spectroscopy (XPS) were obtained by using K-Alpha+. Dynamic light scattering (DLS) measurement was performed by NanoBrook ZetaPlus. Confocal images were taken using a Leica TSC SP8 confocal laser scanning microscope (Germany) with a $63 \times$ oil-immersion objective.

Calculation of the photothermal conversion efficiency. The photothermal conversion efficiency of MSbNSs was determined according to a previously reported method as follows Equation. ${ }^{43}$

$$
\eta=\frac{h A \Delta T-Q_{s}}{I\left(1-10^{-A_{\lambda}}\right)}
$$

Where $h$ is the coefficient of heat transfer, $\mathrm{A}$ is the container surface area, $\Delta T$ is the temperature change of the PEGylated MSbNSs-3 solution, / is the power density of the NIR laser, $A_{\lambda}$ is the absorbance of the solution of PEGylated MSbNSs-3 at $1210 \mathrm{~nm}$, and $Q_{s}$ is the heat associated with the light absorbance of the water.

\section{Declarations}

\section{Acknowledgements}

This work was partially supported by the National Natural Science Foundation of China (61620106016/ 61835009/ 61775145/61005172/ 61805158); Guangdong Province Key Area R\&D Program (2019B110233004); and Shenzhen Fundamental Research Project (JCYJ20190808160207366, JCYJ20190808114609361). The authors wish to acknowledge the assistance on HRTEM observation received from the Electron Microscope Center of the Shenzhen University. All animal experiments were performed under the regulations of Animal Ethical and Welfare Committee of Shenzhen University (AEWC-SZU).

\section{Author contributions}

J.L.Q. and J.S. guided the overall research project. Y.C. conceived the idea, developed the mesoporous antimony nanospheres growth method, and carried out most of experiments and measurements. Z.Y. was involved in the analysis of all data and co-wrote the manuscript. Y.G.R. and C.B.L. contributes to the 
photoacoustic image. M.W., Q. W., Z.F.F., L.W.L. analyzed the data in part. K.Z. contributes to the FDTD measurements. All authors have approved the final version of the manuscript.

\section{Additional information}

Supplementary information accompanies this paper at https://

\section{Competing interests}

The authors declare no competing interests.

\section{References}

1. Singh G, et al. Emerging trends in porous materials for $\mathrm{CO} 2$ capture and conversion. Chem. Soc. Rev. 49, 4360-4404 (2020).

2. Zhao T, Elzatahry A, Li X, Zhao D. Single-micelle-directed synthesis of mesoporous materials. Nat. Rev. Mater. 4, 775-791 (2019).

3. Yan Y, et al. Mesoporous Nanoarchitectures for Electrochemical Energy Conversion and Storage. Adv. Mater. 32, 2004654 (2020).

4. Zhang Y, et al. Ultrasmall-Superbright Neodymium-Upconversion Nanoparticles via Energy Migration Manipulation and Lattice Modification: 808 nm-Activated Drug Release. ACS Nano 11, 2846-2857 (2017).

5. Lin H, Chen Y, Shi J. Nanoparticle-triggered in situ catalytic chemical reactions for tumour-specific therapy. Chem. Soc. Rev. 47, 1938-1958 (2018).

6. Lan K, et al. Stable Ti3+ Defects in Oriented Mesoporous Titania Frameworks for Efficient Photocatalysis. Angew. Chem. Int. Ed. 59, 17676-17683 (2020).

7. Chen Y, et al. Virus-Inspired Deformable Mesoporous Nanocomposites for High Efficiency Drug Delivery. Small 16, e1906028 (2020).

8. Ren Y, et al. Synthesis of orthogonally assembled 3D cross-stacked metal oxide semiconducting nanowires. Nat. Mater. 19, 203-211 (2020)..

9. Pan L, et al. Nuclear-Targeted Drug Delivery of TAT Peptide-Conjugated Monodisperse Mesoporous Silica Nanoparticles. J. Am. Chem. Soc. 134, 5722-5725 (2012)..

10. Wang W, et al. Engine-Trailer-Structured Nanotrucks for Efficient Nano-Bio Interactions and Bioimaging-Guided Drug Delivery. Chem 6, 1097-1112 (2020).

11. Chen Y, Yu M, Ye S, Song J, Qu J. All-inorganic CsPbBr3 perovskite quantum dots embedded in dualmesoporous silica with moisture resistance for two-photon-pumped plasmonic nanoLasers. Nanoscale 10, 6704-6711 (2018).

12. Yu Z, et al. Generating New Cross Relaxation Pathways by Coating Prussian Blue on NaNdF4 for Enhanced Photothermal Agents. Angew. Chem. Int. Ed. 58, 8536-8540 (2019). 
13. Chen J, Zhu Y, Wu C, Shi J. Nanoplatform-based cascade engineering for cancer therapy. Chem. Soc. Rev. 49, 9057-9094 (2020).

14. Iqbal M, et al. Continuous Mesoporous Pd Films by Electrochemical Deposition in Nonionic Micellar Solution. Chem. Mater. 29, 6405-6413 (2017).

15. Lim H, et al. A universal approach for the synthesis of mesoporous gold, palladium and platinum films for applications in electrocatalysis. Nat. Protoc. 15, 2980-3008 (2020).

16. Yang $\mathrm{H}$, et al. Selective $\mathrm{CO}_{2}$ Reduction on 2D Mesoporous Bi Nanosheets. Adv. Energy. Mater. 8 , 1801536 (2018).

17. Zhang M, Zhang L, Chen Y, Li L, Su Z, Wang C. Precise synthesis of unique polydopamine/mesoporous calcium phosphate hollow Janus nanoparticles for imaging-guided chemo-photothermal synergistic therapy. Chem. Sci. 8, 8067-8077 (2017).

18. Zhou Y, et al. Mesoporous PdAg Nanospheres for Stable Electrochemical CO2 Reduction to Formate. Adv. Mater. 32, 2000992 (2020).

19. Jiang B, et al. Mesoporous Metallic Iridium Nanosheets. J. Am. Chem. Soc. 140, 12434-12441 (2018).

20. Jiang B, et al. Mesoporous metallic rhodium nanoparticles. Nat. Commun. 8, 15581 (2017).

21. Lv H, et al. Asymmetric Multimetallic Mesoporous Nanospheres. Nano Lett 19, 3379-3385 (2019).

22. Xu Y, et al. Trimetallic PtPdCo mesoporous nanopolyhedra with hollow cavities. Nanoscale 11, 47814787 (2019).

23. Malgras V, et al. Nanoarchitectures for Mesoporous Metals. Adv. Mater. 28, 993-1010 (2016).

24. Wang $\mathrm{H}$, et al. Shape- and Size-Controlled Synthesis in Hard Templates: Sophisticated Chemical Reduction for Mesoporous Monocrystalline Platinum Nanoparticles. J. Am. Chem. Soc. 133, 1452614529 (2011).

25. Huang $X$, et al. A Facile Strategy to Pt3Ni Nanocrystals with Highly Porous Features as an Enhanced Oxygen Reduction Reaction Catalyst. Adv. Mater. 25, 2974-2979 (2013).

26. Chen S, Su H, Wang Y, Wu W, Zeng J. Size-controlled synthesis of platinum-copper hierarchical trigonal bipyramid nanoframes. Angew. Chem. Int. Ed. 54, 108-113 (2015).

27. Pramanik M, Tominaka S, Wang ZL, Takei T, Yamauchi Y. Mesoporous Semimetallic Conductors: Structural and Electronic Properties of Cobalt Phosphide Systems. Angew. Chem. Int. Ed. 56, 1350813512 (2017).

28. Li W, Rong P, Yang K, Huang P, Sun K, Chen X. Semimetal nanomaterials of antimony as highly efficient agent for photoacoustic imaging and photothermal therapy. Biomaterials 45, 18-26 (2015).

29. Yu X, Liu X, Yang K, Chen X, Li W. Pnictogen Semimetal (Sb, Bi)-Based Nanomaterials for Cancer Imaging and Therapy: A Materials Perspective. ACS Nano, 15, 2038-2067 (2021).

30. Pumera M, Sofer Z. 2D Monoelemental Arsenene, Antimonene, and Bismuthene: Beyond Black Phosphorus. Adv. Mater. 29, 1605299 (2017). 
31. Peng L, Ye S, Song J, Qu J. Solution-Phase Synthesis of Few-Layer Hexagonal Antimonene Nanosheets via Anisotropic Growth. Angew. Chem. Int. Ed. 58, 9891-9896 (2019).

32. Liu T, et al. Two-Stage Size Decrease and Enhanced Photoacoustic Performance of StimuliResponsive Polymer-Gold Nanorod Assembly for Increased Tumor Penetration. Adv. Funct. Mater. 29, 1806429 (2019).

33. Miao Q, Pu K. Organic Semiconducting Agents for Deep-Tissue Molecular Imaging: Second NearInfrared Fluorescence, Self-Luminescence, and Photoacoustics. Adv. Mater. 30, 1801778 (2018).

34. Yu Z, Chan WK, Zhang Y, Tan TTY. Near-infrared-II activated inorganic photothermal nanomedicines. Biomaterials, 269, 120459 (2020).

35. Chen J, et al. Tocilizumab-Conjugated Polymer Nanoparticles for NIR-II Photoacoustic-ImagingGuided Therapy of Rheumatoid Arthritis. Adv. Mater. 32, 2003399 (2020).

36. Yu Z, Chan WK, Tan TTY. Neodymium-Sensitized Nanoconstructs for Near-Infrared Enabled Photomedicine. Small 16, 1905265 (2020).

37. Chen Y, et al. Antimony Nanopolyhedrons with Tunable Localized Surface Plasmon Resonances for Highly Effective Photoacoustic-Imaging-Guided Synergistic Photothermal/Immunotherapy. Adv. Mater. 33, 2100039 (2021).

38. Lu G, et al. Antimonene with two-orders-of-magnitude improved stability for high-performance cancer theranostics. Chem. Sci. 10, 4847-4853 (2019).

39. Tao W, et al. Antimonene Quantum Dots: Synthesis and Application as Near-Infrared Photothermal Agents for Effective Cancer Therapy. Angew. Chem. Int. Ed. 56, 11896-11900 (2017).

40. Tao W, et al. Two-Dimensional Antimonene-Based Photonic Nanomedicine for Cancer Theranostics. Adv. Mater. 30, 1802061 (2018).

41. Moshchenko SP, Lyamkina AA. Modelling-the-localized-surface-plasmon-resonance-of-nanoclustersof-group-iii-metals-and-semimetallic-antimony. J. Sib. Fed. Univ. Math. Phys. 7, 211-217 (2014).

42. Dibaba ST, et al. NIR Light-Degradable Antimony Nanoparticle-Based Drug-Delivery Nanosystem for Synergistic Chemo-Photothermal Therapy in Vitro. ACS Appl. Mater. Inter. 11, 48290-48299 (2019).

43. Li B, et al. Cu7.2S4 nanocrystals: a novel photothermal agent with a $56.7 \%$ photothermal conversion efficiency for photothermal therapy of cancer cells. Nanoscale 6, 3274-3282 (2014).

\section{Figures}



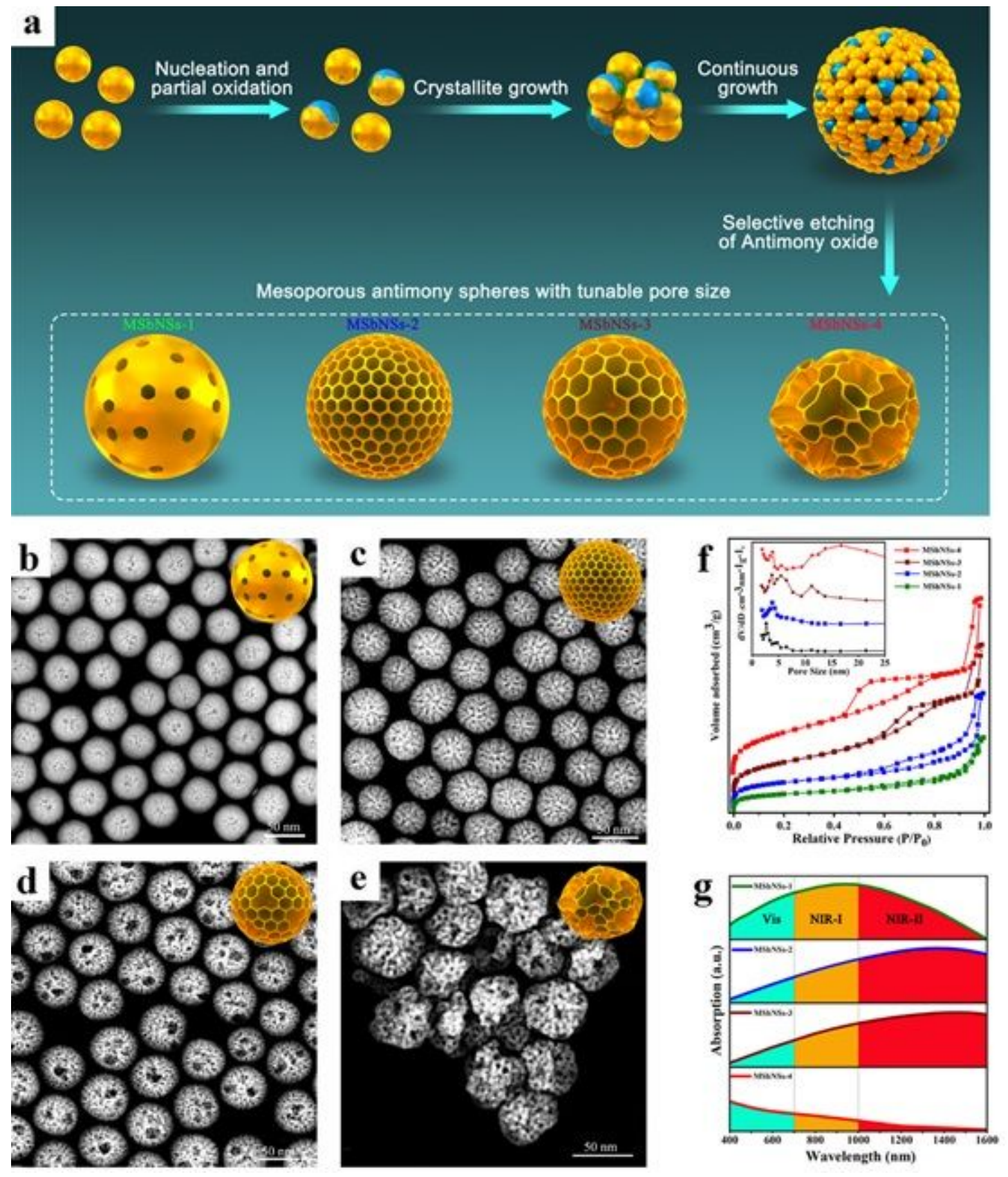

Figure 1

Fabrication and characterization of MSbNSs. a Schematic illustration of the fabrication of MSbNSs with tunable pore sizes. STEM images of b MSbNSs-1, c MSbNSs-2, d MSbNSs-3, e MSbNSs-4. f N2 physisorption isotherms and their corresponding pore size calculated by the BJH method. $\mathrm{g}$ Vis-NIR absorption spectra of MSbNSs-1/2/3/4. 



Figure 2

Formation mechanism study of MSbNSs. a XRD patterns, b XPS analysis of MSbNSs-1/2/3/4. c Stepoxidization reaction mechanism of MSbNSs. $d$ FTIR spectra of MSbNSs-1/2/3/4. e EDS mapping of MSbNSs-3. $f$ The change of residual ratios of $O$ and $S$ elements in MSbNSs-1/2/3/4, respectively.
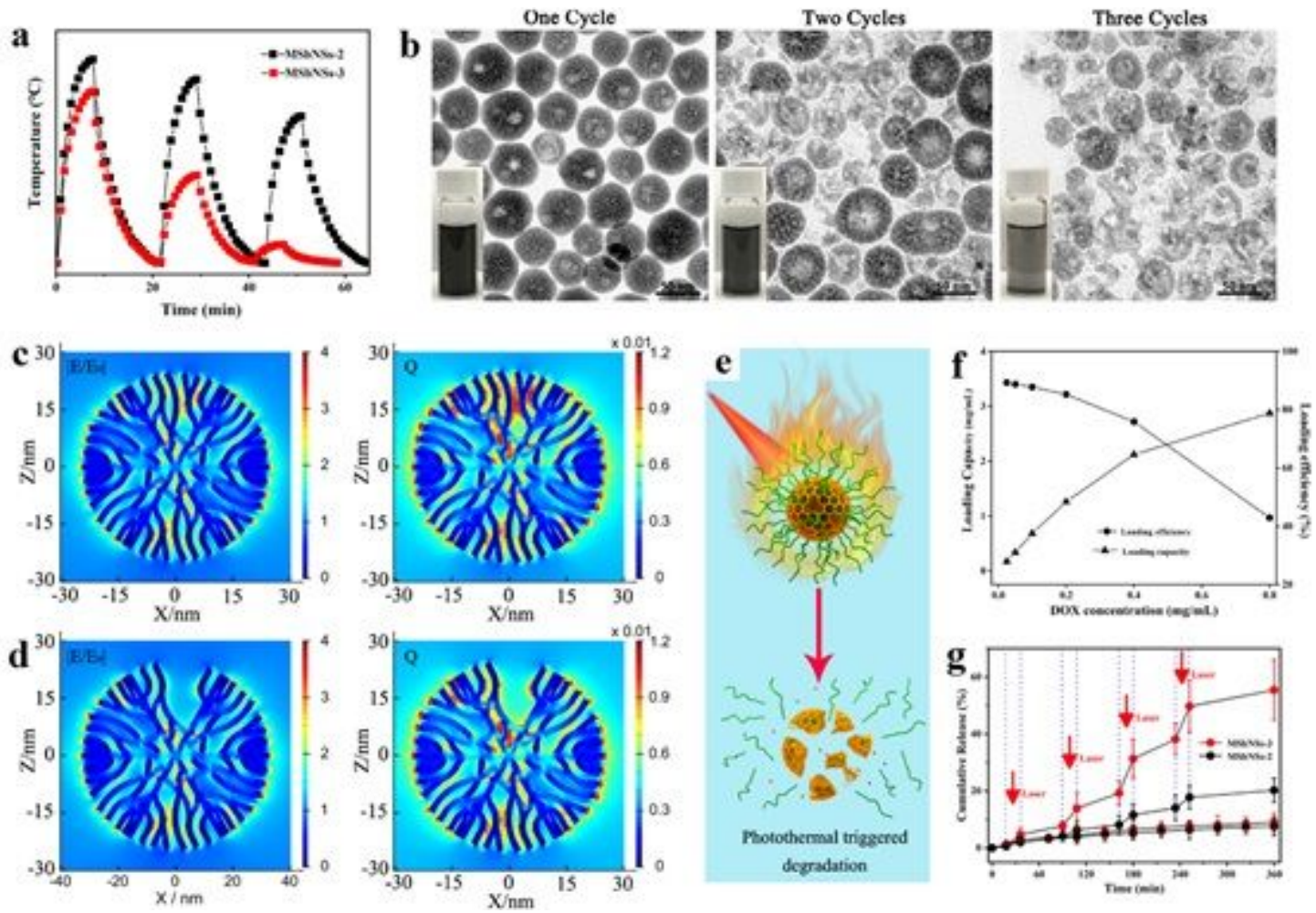

\section{Figure 3}

Photothermal degradation and drug loading/release of MSbNSs. a Photothermal stability study of PEGylated MSbNSs-2/MSbNSs-3 under the irradiation of $1210 \mathrm{~nm}$. b TEM images of PEGylated MSbNSs- 
3 after different heating and cooling cycles. Simulation results of the electric field enhancement (|E/EO|) and the heat power density Q inside the c MSbNSs-2 and d MSbNSs-3 illuminated by $1210 \mathrm{~nm}$ light. e Schematic illustration of photothermal triggered degradation and DOX release. $\mathrm{f}$ DOX loading capacity and efficiency of MSbNSs-3. g Photothermal triggered DOX release in PBS ( $\mathrm{pH}=7.4)$ irradiated with $1 \mathrm{~W}$ $\mathrm{cm}-2$ of a $1210 \mathrm{~nm}$ laser.

a
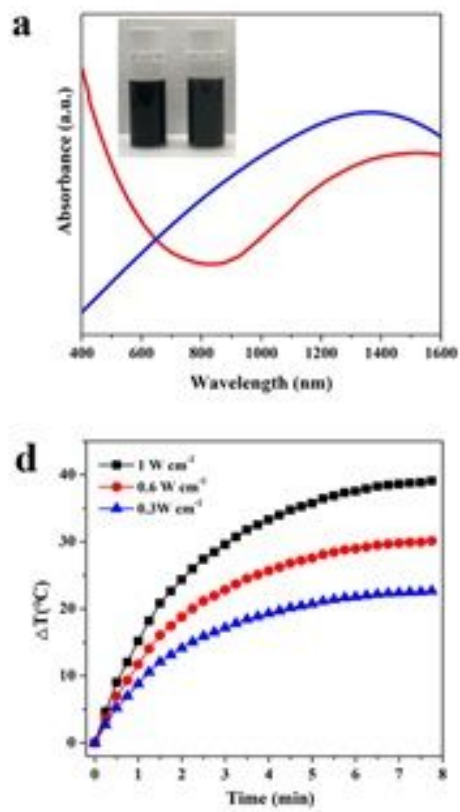
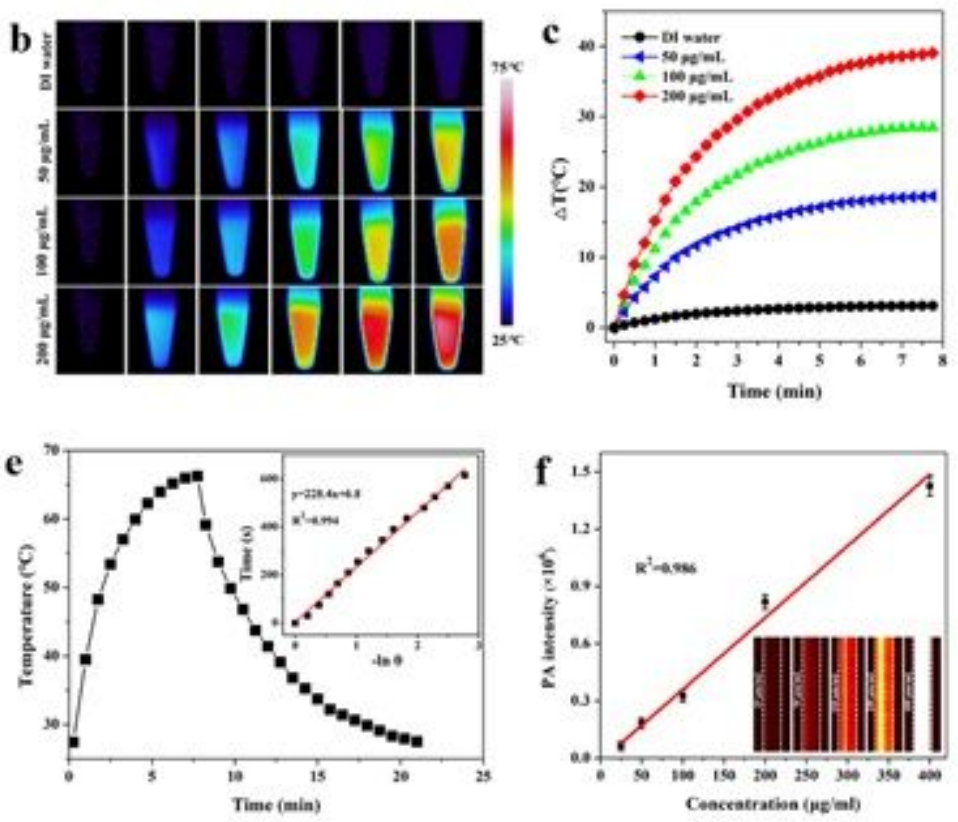

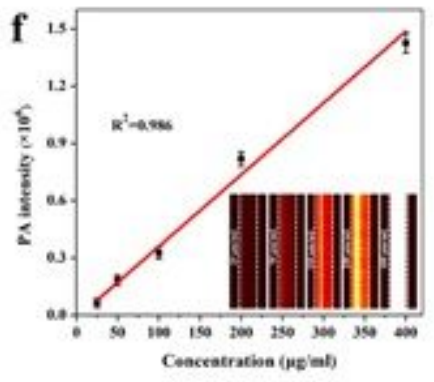

Figure 4

NIR-II photothermal performance of PEGylated MSbNSs-3. a Vis-NIR spectra of MSbNSs-3 before (blue line) and after (red line) PEG modification. b Photothermal images and c temperature change curves of the PEGylated MSbNSs-3 with different concentrations under $1210 \mathrm{~nm}$ laser irradiation for about $8 \mathrm{~min}$. $\mathrm{d}$ Temperature curves of the PEGylated MSbNSs-3 under $1210 \mathrm{~nm}$ laser irradiation at different power densities for about 8 min. e The photothermal heating curve of the PEGylated MSbNSs-3 under laser irradiation (1210 nm, 1W cm-2) and cooling curve after turning off the laser. The inset shows the linear relationship between $-\ln \theta$ and time obtained from the cooling. $f$ Photoacoustic amplitudes at $1210 \mathrm{~nm}$ as a function of concentrations of the PEGylated MSbNSs-3. 

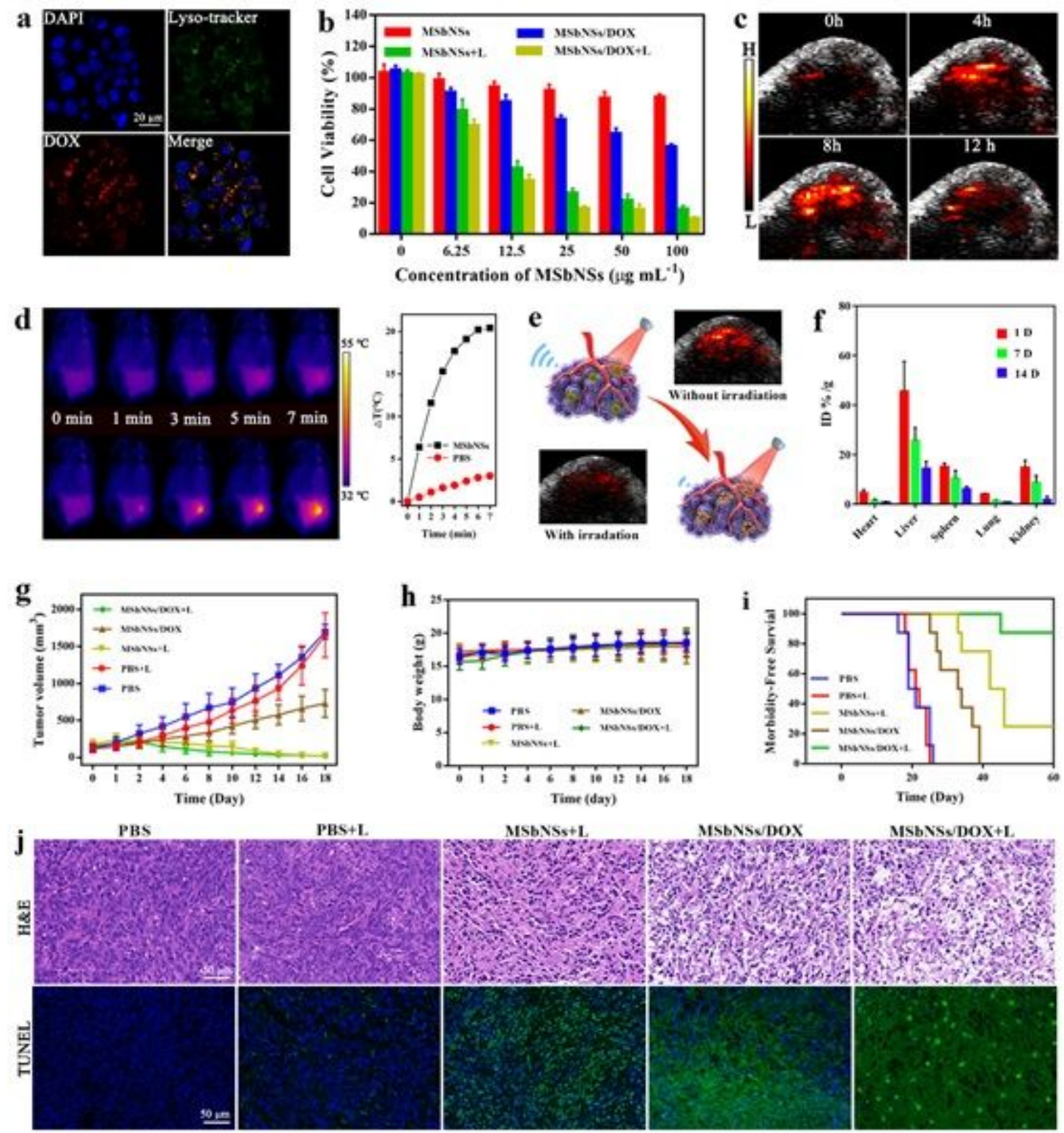

Figure 5

In vitro and in vivo synergistic chemotherapy and NIR-II PTT via PEGylated MSbNSs-3/DOX. a Confocal microscopy images of panc02 cancer cells to show the internalization of PEGylated MSbNSs-3/DOX. b Cell viability of panc02 cells treated with PEGylated MSbNSs-3, PEGylated MSbNSs-3 + laser, PEGylated MSbNSs/DOX, and PEGylated MSbNSs-3/DOX + laser. c PA images showing changes in PA signal (red) over time with PEGylated MSbNSs-3/DOX being injected at the tumor site. $d$ IR thermal images of panc02-tumor mice injected with PBS and PEGylated MSbNSs-3 in response to 1210 laser irradiation. e Schematic illustration of in vivo photothermal triggered degradation and the change of PA images before and after irradiation for 15 min. f Biodistribution of PEGylated MSbNSs-3. The error bars represent standard deviations $(n=3)$.g Tumor growth curves, $h$ body weight, and i survival proportions of mice after systemic treatment with PBS, PBS + laser, PEGylated MSbNSs-3 + laser, PEGylated MSbNSs-3/DOX, and PEGylated MSbNSs-3/DOX + laser $(n=6)$. j H\&E and TUNEL staining for tumor acquired $24 \mathrm{~h}$ after systemic treatment. 


\section{Supplementary Files}

This is a list of supplementary files associated with this preprint. Click to download.

- Supportinginformations.docx 